\title{
Actividad de Renina Plasmática (ARP) en Niños Normales y Desnutridos
}

\author{
Dres.: Carlos Saieh A. ${ }^{1}$, Leonardo Acuña O. de $Z^{2}$ Matilde Maddaleno H. ${ }^{2}$, \\ Sres.: Gilberto López $\mathbf{S}^{3}$, Victoria Idalzoaga P. $^{3}$.
}

Plasma Renin activity in normal and malnourished children

\begin{abstract}
Plasma renin activity (PRA) was measured by radioimmunoassay in 66 children, aged 1 month to 15 years. 25 of them suffered malnutrition.

No differences in PRA were found to exist between sexes. PRA varied inversely with sodium intake and a progresive decrease of PRA with age was demostrated: The values obtained fluctuated from $7.30 \pm 3.39 \mathrm{mg} \mathrm{x} \mathrm{ml} \mathrm{xh}$ at 1 to 5 months to $3.34 \pm 2.11 \mathrm{mg} \mathrm{x} \mathrm{ml} \times \mathrm{xh}$. at 10 to 14 years of age.

In this work no statistic differences were found between normal and malnourished children.
\end{abstract}

La renina es una enzima proteolitica sintetizada, almacenada y secretada por el aparato yuxtaglomerular de los rinones y también en órganos como el útero, placenta, vasos sanguíneos, cerebro, suprarrenales, etc. ${ }^{1-2 \cdot 3 \cdot 4 \cdot 5-6}$. Esta sustancia actua sobre un sustrato, el angiotensinógeno, transformándolo en angiotensina $\mathrm{I}$, el que rápidamente es transformado en angiotensina II por acción de una enzima convertidora, que se encuentra principalmente en el pulmón, aunque también se ha encontrado en otros órganos ${ }^{7}$. La angiotensina II es el más potente agente vasocontrictor conocido y tiene una vida media circulante menor de un minuto; esta es rápidamente degradada a angiotensina III por varias angiotensinasas encontradas en el plasma y otros tejiodos ${ }^{8}$. El rol exacto de esta sustancia no está totalmente aclarado hasta la fecha.

Numerosas publicaciones ${ }^{9}-10 \cdot 11-12 \cdot 13-$ 14 - 15 destacan la importancia de este sistema en

1 Unidad de Nefrología. Hospital Luis Calvo Mackenna.

${ }^{2}$ Becados Servicio de Pediatría. Hospital Luis Calvo Mackenna.

3 Medicina Nuclear. Universidad Católica de Chile pacientes cuya hipertensión está relacionada de alguna manera con patología que se caracteriza por inducir una baja perfusión en uno o ambos riñones.

La interpretación de la ARP en nuestro medio se dificulta por carecer de datos sobre normalidad, aunque en el extranjero existen trabajos que sugieren una disminución progresiva de los niveles desde el período de recién nacido hasta la adolescencia ${ }^{11}$ $-16-17-1 B$

Nuestro propósito es conocer los valores normales de ARP en niños normales y en desnutridos en diferentes grupos etarios, tomando las muestras en las condiciones habituales en nuestro medio.

\section{MATERIAL Y METODOS}

Se estudiaron 102 nifios controlados en el Hospital Luis Calvo Mackenna durante los meses de Marzo de 1981 a Marzo de 1982, de los cuales 66 cumplieron con los requisitos mínimos, es decir:

Durante los 3 dias previos a la toma de la muestra no haber recibido ningún medicamento o soluciones parenterales y haber mantenido una dieta con ingesta de electrolitos invariable $y$ aportes nor- 
males de todos ellos. Mediante anamnesis, examen físico, examen de orina completa, urocultivo, hemograma, electrolitos plasmáticos y en orina $y$ creatininemia se descartó patologias que pudieran influenciar la ARP.

Veinticinco niños de los 66 eran desnutridos, sin patología asociada. Los 41 niños restantes eran eutróficos que habían consultado por trastornos respiratorios agudos y que en el momento del estudio no presentaron manifestaciones clínicas ni de laboratorio de la enfermedad y tampoco recibían tratamiento.

Las muestras de siangre fueron obtenidas luego de un periodo de reposo absoluto de por lo menos 12 horas, desde vena periférica entre las 09 y las 11 horas A.M., con los niños en decúbito dorsal. En los pre--escolares y escolares se usaron las venas del pliegue del codo y en los lactantes la yugular.

\section{METODO}

Reactivos: estandar de angiotensina humana; antisuero angiotensina I producido en conejo; angiotensina 1 marcada con $\mathrm{I}^{125}$, solución de 8 . hidroxiquinoleina, dimercaprol y tampón de ensayo TRIS acetato O. $1 \mathrm{M} \mathrm{pH} 7.4$ obtenidos de New England Nuclear; $\mathrm{Na}_{2}$ EDTA - adquirido desde Merck Sharp y Dome.

La sangre fue extraída $y$ luego vertida en tubo de vidrio yue contenía una cantidad de Na EDTA suficiente para obtener una concentración de 1 $\mathrm{mg} / \mathrm{ml}$ en sangre. Se separó el plasma en centrífuga refrigerada a $4^{\circ} \mathrm{C}$ por $10 \mathrm{~min}$, a $3.000 \mathrm{rpm}$. y se congeló a $-20^{\circ} \mathrm{C}^{3}$.

La técnica para medir ARP incluye una incubación de las muestras por 1 hora a $37^{\circ} \mathrm{C}$ y un período de incubación similar pero a $4^{\circ} \mathrm{C}$ para control de las mismas muestras. Las condiciones de incubación del ensayo permiten la generación de angiotensina I por la renina plasmática en un sistema tampón a pH óptimo de 5.5 a 6.0 en presencia de inhibidores enzimáticos para la angiotensina proteolítica y para la enzima convertidora de angiotensina $[\text { a angiotensina }]^{5-6}$.

Al final del período de incubación la angiotensina I generada fue medida por radioinmunoensayo.

La actividad de la renina fue calculada sustrayendo el valor de la muestra control $\left(4^{\circ} \mathrm{C}\right)$ del valor obtenido a $370 \mathrm{C}$ y se expresa como nanogramos (ng) de angiotensina l producidas por ml. de plasma por hora.

\section{RESULTADOS}

La Tabla 1 muestra una distribucjón semejante de edades para ambos sexos y la ausencia de dife- rencias en los valores de ARP entre hombres y mujeres.

Tabla 1 .

Edad Sexo y ARP

\begin{tabular}{|c|c|c|c|c|c|c|}
\hline \multirow[b]{2}{*}{$\begin{array}{l}\text { EDAD } \\
\text { (aగos) }\end{array}$} & \multicolumn{3}{|c|}{ HOMBRES } & \multicolumn{2}{|c|}{ MUJERES } & \multirow[b]{2}{*}{ \pm D. $s$} \\
\hline & $\underset{(n g x}{n}$ & $\begin{array}{l}\text { RP* } \\
m(x h)\end{array}$ & tD.s. & $\mathbf{n}$ & ARP* & \\
\hline $0 \quad 4$ & 25 & 5.25 & 1.78 & 22 & 5.86 & 3.00 \\
\hline 5. 9 & 4 & 2.57 & 2.09 & 4 & 4.06 & 2.26 \\
\hline $10 \cdot 14$ & 5 & 3.52 & 2.21 & 5 & 3.24 & 2.22 \\
\hline
\end{tabular}

El aporte diario de sodio en los nifos, durante el estudio, se presenta en la Tabla 2. Todos ellos tuvieron valores de electrolitos en plasma dentro de limites normales $(139 \pm 2.6 \mathrm{mEq} / \mathrm{lt}$.). Cabe hacer notar que entre los 3 y 4 años de edad y de ahí en adelante, la cantidad y calidad de la alimentación se mantiene sin grandes variaciones a las distintas edades, $y$ eso hace que el sodio recibido sea similar en cantidad a lo estimado en los adultos, vale decir que a partir de los 20 kilos de peso corporal un niño recibe aproximadamente 170 $\mathrm{mEq}$. de sodio por día, según la dieta chilena habitual.

Tabla 2.

Edad e Ingesta de Sodio

$\begin{array}{lcc}\text { EDAD } & \mathrm{X}(\mathrm{mEq} \times \mathrm{K}) & \pm \mathrm{D} . \mathrm{S} . \\ 0 \quad 5 \text { Meses } & 2.96 & 1.72 \\ 6-\text { 11 Meses } & 2.75 & 0.92 \\ 1-2 \text { Años } & 2.65 & 0.75 \\ 3 \text { 4 Años } & 8.12 & 2.08 \\ 5 \text { Años } & 8.77 & 2.39\end{array}$

La Tabla 3 muestra la distribución por edad y los valores de ARP en los distintos grupos etarios. Se aprecia que el valor de ARP fue decreciendo a medida que aumenta la edad.

En la Tabla 4 se compara los valores de ARP a distintas edades en los niños eutróficos y desnutridos. No se encontró diferencias estadísticas significativas en ambos grupos (p. 0.05).

La Figura 1 muestra las variaciones experimentadas por la ARP en relación a la ingesta de $\mathrm{Na}$, existiendo una relación inversá. 
Tabla 3.

Edad y ARP

$\begin{array}{lrrr}\text { EDAD } & n & \begin{array}{c}\text { ARP } \pm \text { D. S. } \\ (\mathbf{n g} \times \mathrm{ml} \times \mathbf{b})\end{array} \\ 0-5 \text { Meses } & 12 & 7.30 & 3.39 \\ 6 \quad 11 \text { Meses } & 5 & 7.30 & 2.42 \\ 1-2 \text { Años } & 20 & 5.46 & 2.73 \\ 3-4 \text { Años } & 10 & 4.40 & 2.67 \\ 5-9 \text { Años } & 8 & 3.98 & 2.26 \\ 10-14 \text { Años } & 11 & 3.34 & 2.11\end{array}$

Tabla 4.

Edad, Estado Nutritivo y ARP

\section{NORMALES}

\section{DESNUTRIDOS}

\begin{tabular}{|c|c|c|c|c|c|c|}
\hline EDAD & & \multicolumn{2}{|c|}{$\begin{array}{c}\text { ARP } \pm D . S \\
(m g \times m l \times h)\end{array}$} & \multirow{2}{*}{$\begin{array}{l}n \\
6\end{array}$} & \multicolumn{2}{|c|}{$\begin{array}{l}\text { ARP } \pm \text { D. S. } \\
(m g \times m i \times h)\end{array}$} \\
\hline 11 & 11 & 7.45 & 2.00 & & 7.15 & 4.58 \\
\hline $2 \mathrm{a}$ & 12 & 4.55 & 1.88 & 8 & 6.21 & $3.6 ?$ \\
\hline$-14 a$ & 18 & 3.51 & 2.09 & 11 & 4.10 & 2.19 \\
\hline . & & & & & & \\
\hline
\end{tabular}

A.R.P. $(\mathrm{ng} / \mathrm{ml} / \mathrm{h})$

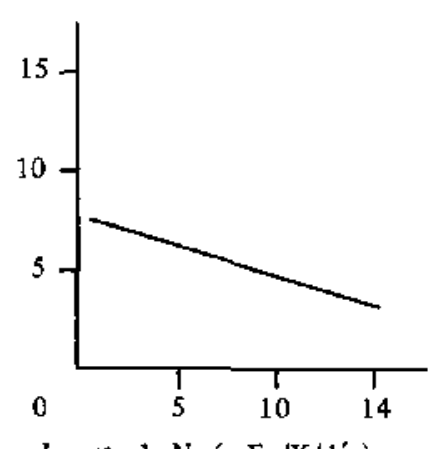

Ingesta de $\mathrm{Na}(\mathrm{mEq} / \mathrm{K} / \mathrm{d}$ ís)

Figura 1.

Actividad de renina plasmática (A.R.P.)

VS ingesta sodio

\section{DISCUSION}

Al analizar nuestros resultados no encontramos diferencias con los trabajos publicados por otros autores en relación a que los valores de ARP disminuyen con la edad, que no hay diferencias en cuanto al sexo y que existe una estrecha correlación con la ingesta de sodio y la ARP10 - 17 - 18 - 19

Llama la atención que nuestros valores de ARP en los diferentes grupos etarios sean más altos que en la mayoría de las series publicadas, y si bien gran parte de las muestras fueron obtenidas en niños con procesos bronquiales en resolución, no encontramos explicación para estas diferencias, excepto yue las condiciones generales del medio, como la sala, el material a usar y el manejo del pa. ciente por el médico y la enfermera no sean hos más adecuados. Las condiciones ideales habria que lograrlas mediante sedación e instalación de un cateter para obtener muestras durante el sueño, lo cual resulta poco práctico. La dispersión observada puede restringirse siendo más estrictos en torno a obtener las muestras en condiciones más cercanas a la ideal.

Los valores de ARP elevados en el recién nacido pueden explicarse debido a que en este período la reabsorción fraccional del sodio está reducida en comparación con el niño mayor y el adulto, lo que lleva a niveles más bajos de sodio pkasmático, esta hiponatremia relativa explica la liberación de angiotensina II "In situ" cuyo efecto sería la disminución del flujo plasmático renal y de la filtración glomerular ${ }^{20}$. Ultimamente se ha demostrado que el sistema renina - angiotensina - aldosterona parece tener un importante papel en el neonato, al regular el balance de agua a través de prostaglandinas renales. Joppich y col. demostraron valores de $\mathrm{PGE}_{2}$ más bajos en recién nacidos de pre término en comparación con los de término. Esta prostaglandina tiene una acción antirrenina, antihormona antidiurética y anti-noradrenalina ${ }^{21}$.

Los valores de ARP van disminuyendo hasta los 4 o 5 años donde se obtienen iguales cifras que las esperadas para el adulto. Estos valores tienen una estrecha correlación con la ingesta de sodio (Tabla 2), hecho fundamental para interpretar correctamente este examen dado que un aumento en la ingesta significará un valor más bajo de ARP y lo inverso sucedera si el paciente ha recibido diuréticos. En otras palabras, condiciones estandar de toma de muestra para ARP significarán conocer perfectamente el aporte de electrolitos y que estos sean constantes en cantidad en los días previos a la toma.

En relación a los nifios desnutridos, sabemos que existe en alguna medida una disminución de la velocidad de filtración glomerular, con un menor flujo plasmático y una hiponatremia leve. ${ }^{22} \cdot 23 \cdot$ 24 , lo que nos hizo suponer que los valores de ARP en niños desnutridos serian diferentes a los eutróficos, pero, como ya se analizó en la Tabla 4, no existen diferencias estadísticas significativas entre ambos grupos. La explicación puede ser que a pesar de los cambios en función renal, volemia y electrolitos presentes en los desnutridos, estos no están lo suficientemente alterados como para dese- 
quilibrar los mecanismos del sistema renina angiotensina. Asi por ejemplo Brown y col. ${ }^{25}$ demostraton que la caida en la concentración de sodio no siempre produce elevación de la ARP, sino sólo cuando los niveles de sodio plasmático están por debajo de $137 \mathrm{mEq} / \mathrm{lt}$. En otras palabras el hecho que no se haya encontrado diferencias entre los valores de ARP de los desnutridos y eutróficos significa solamente que los cambios producidos en los desnutridos en relación a volemia, electrolitemia, etc., no han sido en este caso, tan importante como para alterar el sistema. Lo anterior nos hace reforzar el planteamiento que el estudio de un niffo desnutrido hipertenso debe considerar, además de los requisitos habituales una valorización precisa de la función renal y electrolitos plasmáticos como se recomienda habitualmente.

\section{RESUMEN}

Se midió la ARP en 66 niños de 1 mes a 15 años de edad, mediante radio-inmuno-ensayo. En el grupo habian 25 sujetos. que sufrían desnutrición. No se encontraron diferencias entre ambos sexos ni entre sujetos normales y desnutridos. La ARP varió inversamente con la ingestión de sodio y mostró una disminución progresiva con la edad.

\section{REFERENCIAS}

1 Jay N. Cohn: Paroxysmal hipertension and hypovolemia N. Eng. J. Med. 275: 643, 1966.

2 David H.P. Streeten, Felix E. Schetter Studies of the Renin-Angiotensin-Aldosterone System in patient with Hypertension and in normal subjets. Am. J. Med. 46: $844,1969$.

3 E. D. Vaughan. R. C. Siveet Peripheral renin and blood presure changes changes following complete unilateral uretal occlusion. J. Jiol. 104: 89, 1970.

4 Best J. B. Coghian J. P., Bett J. H. and Gran E. J. Circulating Angiotensin II and Aldosterane levels during dietary sodium restriction. Lancet Dec. 18: 1353,1971 .

S Ryart J. W., Smith V, and Niemo yer R.S. Angiotensin I: Metabolism by plasma membrane of lungs. Science 176: 63, 1972 .

6 Overtuf $M .$. Fitz $A$, and Mc. Bride J. Renin activity, sodium and potassium in hypertensive paticnts. J. Chron. Disease 25: 621, 1972.

${ }^{7}$ Freedlender A. E. Goodfrien T. L. Renin and the angiotensins. Methods of Hormones radioimmune assay. 2nd. Ed. Academic Press Inc. Pag. 889, 1979.

"Catt. 3 . Doughs $I$, in "Nucleat modicine in Vitro". Ch. 21 Lippincott Co. Philadelphia 1974.

9 Robert C. Tarazt. Renir angiotensin and Cardiac lesions J. of Lab, and Clin. Med. 95: 155, 1980.

10 J. R. Stockigt. C. A. Nookes Renal - vein tenin in various forms of renal hypertension. Lancet June: $1 \mathrm{~J} 94,1972$

11 Dillon $M . J$. Renin and hypertension in childhood. Arch. Dis. Child, 49:831, 1974.

12 Richard H. White, M. Schambelans, Нypertension, hyperreninemia and secondary hypcraldosteronim in systemic necrotizing vasculitis. Ann. Int. Med. 92:199, 1980 .

13 Cyrus Akrami, M. D. Bonita Falkner Plasma renin and accurrence of hypertension in children with burn injuries. J. Trauma 20: 130, 1980.

14 Lieberman $J$, and Rea $r, H$. Serum angiotensin converting enzymes in leprosy and coccididomycosis Asn. Intern. Med. 87: 422, 1977.

15 Rohatgy P. E., Massey T. H, and Ryan $J$. W. Serum Angiotensin converting enzyme and sarcoidosis. Clin. Research 26: 62A, 1978.

16 Anita Aperia, Ove Broberger Renal tesponse to an otal sodium load in newborn full term infants. Act. Pediat. Scand. 61: 670, 1972 .

17 Hiner L. B., Gruskin A. B., Boluarte H. J. and Cote $M$. Plasma renin activity in normal children. J. Pediat. 89: $258,1976$.

18 Sassard $J$. Sann L., Vincent $R$, and Cier J. F. Plasma renin activity in normal subjets from infancy to puberty. J. Clin. Endocrinol. Metab. 40: 524, 1975.

19 Oparil $S$. Haher $F$. The renin. angiotensin system. N. Ens. J. Med. 291: 389, 1974.

20 Norero C. Algunas consideraciones sobre la patogenia de la insuficiencia renal aguda. Rev. Chil. Ped. 53: 66, 1982.

21 Joppich R., Scherer $B$ and Weber P.C. Renal Prostaglandins. Relationship to the development of Blood pressure and Coneentrating capacity in pre-term and full term healthy infants. Cur. J. Pediatr. 132: 253, 1979 .

22 Nichols B. L. Alverado $J$, and Rodriguez J. Therapeutics implications of elcctrolyte, water and nitrogen losses during recovery from protcin calorie malnutrition. J. Pediatr. B4: 759, 1974.

23 Martner J. A., Jarpa S. y Ariztía A. Estudios en lactantes distróficos. Metabolismo del agua y electrolitos. Rev. Chil. Ped. 20: 121, 1963.

24 Frenk S., Metcff J. Gómez F. et al. Intracellular composition and homeostatic mechanisms in severe chronic infantile malnutrition. Pediatrics 20: 105, 1957.

25 Brown J., Davies D. L., Lever A. F. and Robertson I. f. Rentu and angiotensin. A survey of some aspects. Postgrad, M. J. 42: 1\$3, 1966 